\title{
Juxtafacet Spinal Synovial Cysts
}

\author{
Haitham El-Beltagy Abd El-Kader \\ Neurosurgery, Al-Menoufia University, Al-Menoufia, Egypt
}

\begin{abstract}
Study Design: This was a retrospective study.
Purpose: To study the surgical outcome of synovial cysts of the lumbar spine through posterior laminectomy in combination with transpedicular screw fixation.

Overview of Literature: Synovial cysts of the lumbar spine contribute significantly to narrowing of the spinal canal and lateral thecal sac and nerve root compression. Cysts form as a result of arthrotic disruption of the facet joint, leading to degenerative spondylolisthesis in up to $40 \%$ of patients.

Methods: Retrospective data from 6 patients, treated during the period of March 2007 to February 2011, were analyzed. All preoperative and postoperative manifestations, extension/flexion radiographs, magnetic resonance imaging, and computed tomography records were reviewed. All underwent surgery for synovial cysts with excision and decompression combined with posterior fixation. The result of surgery was evaluated with Macnab's classification. An excellent or good outcome was considered as satisfactory. Japanese Orthopedic Association Scale was used for evaluation of back pain.

Results: All patients included in this study had excellent outcomes as regarding to improvement of all preoperative manifestations and returning to normal daily activities. Only 2 cases developed postoperative transient cerebro-spinal fluid leak and were treated conservatively and improved during the follow up period.

Conclusions: Although this study included a small number of cases and we could not have statistically significant results, the good outcome of decompression of synovial cysts combined with posterior fixation and fusion encouraged us to recommend this approach for patients with juxtafacet synovial cysts.
\end{abstract}

Keywords: Synovial cysts; Posterior decompression; Transpedicular fixation; Fusion

\section{Introduction}

Synovial cysts of the spine are cystic dilatations of the synovial sheaths and may occur in any of the spinal areas: cervical, dorsal or lumbo-sacral region thoracic. Synovial cysts occur more frequently in the lumbar spine, especially at L4-5 level, than in the cervical or thoracic regions. In the study of Marshman et al. [1], (94\%) lumbar synovial cysts were found along with $1(2 \%)$ thoracic and $1(2 \%)$ cervico-thoracic cyst. The "juxtafacet cyst" has been the most common entity of intraspinal cysts and encompasses the synovial cyst and ganglion cyst. Two types of cysts share common symptomatic and radiological characteristics but different histological backgrounds. Therefore, a new name has been introduced to replace the "juxtafacet cyst" since Kao used it first [2]. Regarding the ganglion cyst, many authors used various names including facet cyst, posterior longitudinal ligament (PLL) cyst, disc cyst

Received Apr 25, 2015; Revised May 4, 2015; Accepted May 6, 2015

Corresponding author: Haitham El-Beltagy Abd El-Kader

Neurosurgery, Al-Menoufia University, Al-Menoufia, 11 El Sadat st., Shebin El-kom, Al-Menoufia, Egypt

Tel: +20-100-343-4915, Fax: +20-48-222-1013, E-mail: hithamelbeltagy@yahoo.com 
or ligament flavum (LF) cyst depending on the origin. On the other hand, some authors have used the synovial cyst as a representative of the juxtafacet cysts including the ganglion cyst [3].

Synovial cysts are based on the laxity of the synovial capsule of the facet joints and frequently associate with spinal instability. The ganglion cyst is believed to arise from degenerated ligaments including the PLL and LF in the mobile spine [4]. The cause of synovial cyst is still unclear. However, it underlies spinal instability, facet joint arthropathy and degenerative spondylolisthesis [5].

Of 1,800 lumbar computed tomography (CT) and magnetic resonance (MR) studies performed over an 18-month interval, Onofrio and Mih [6] reported 11 $(0.6 \%)$ instances of synovial cysts involving the lumbar spine. Of 440 cases of synovial cysts reported in 15 series, the smallest study consisted of a single case report, while the largest series presented by Lyons et al. [7] included 194 cases. Lumbar synovial cyst symptoms include unilateral or bilateral radiculopathy, neurogenic claudication typically in those with underlying spinal stenosis, neurological deficits including motor deficits, sensory deficits and reflex abnormalities.

Conservative treatment modalities include bed rest, orthopedic corsets, CT-guided needle aspiration, and intraarticular injection of corticosteroid drugs. However, these treatments often show short term or no improvement at all. Surgical treatment is largely recommended in all cases of intractable pain or neurological deficit. The surgical technique will depend on the site, size, and associated other factors of the cysts, for example, duration of symptoms and involvement of surrounding structures, etc. [8].

Lumbar synovial cyst surgery includes unilateral or bilateral laminotomies, hemilaminectomies, or laminectomies alone or in combination with in situ or instrumented fusion. The surgical management or extent of decompression is mandated by the degree of co-existent stenosis. Many studies rely heavily on laminectomy to adequately decompress or excise the synovial cyst and deal with the attendant stenosis while patients with lumbar synovial cysts accompanied by degenerative spondylolisthesis may further benefit from simultaneous fusion [9]. Resection and decompression with fusion remain an appropriate option. It appears that the optimal approach for patients with juxtafacet cysts remains unclear. The best surgical treatment approach for each particular individual would appear to remain speculative [10].

\section{Materials and Methods}

This retrospective study included six patients, 4 males and 2 females (M:F ratio=2:1) between 25 to 55 years old at the time of diagnosis (mean age, 40 years) each presenting with symptomatic lumbar synovial cyst. The patients were admitted and managed at the Department of Neurosurgery, Al-Menoufia University, Egypt between March 2007 and September 2010.

\section{Inclusion criteria}

(1) Symptomatic synovial cyst (sciatica and/or neurogenic claudication) not responding to conservative treatment for at least 3 months. (2) Synovial cyst evident on a magnetic resonance image (MRI). (3) Medically fit (e.g., no bleeding disorders).

Confirmation of diagnosis was done through X-ray radiographs (including dynamic films), CT, and MRI (Figs. 1,2). After selection of patients and obtaining surgical consent, all the patients included in the study underwent decompression with excision of the synovial cyst through posterior laminectomy combined with transpedicular screw fixation and posterolateral fusion (PLF) (Fig. 3). All the possible samples retrieved during surgery (Fig. 4) were sent for histological examination (Fig. 5).

\section{Clinical follow-up}

The clinical symptoms were assessed at 3, 6, and 12 months post discharge from the hospital through the Japanese Orthopedic Association Scale (JOA). The JOA score was determined via direct questioning to assess

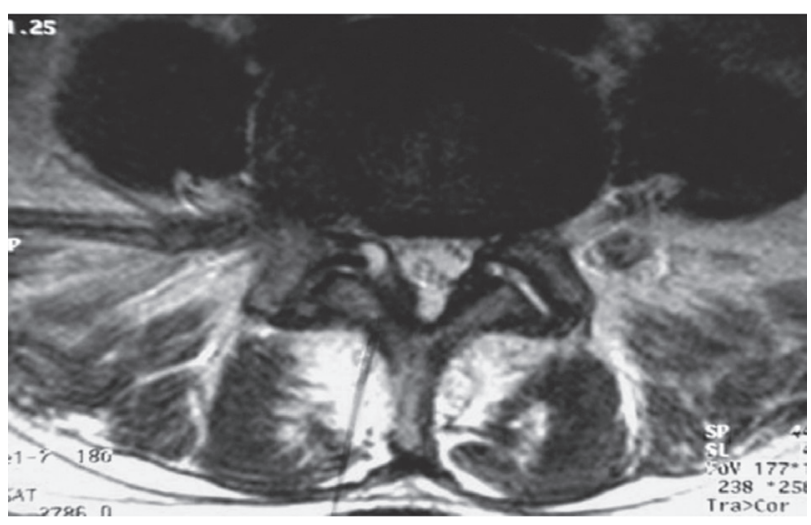

Fig. 1. A preoperative axial magnetic resonance imaging scan showing a right $L 4-5$ synovial cyst. 
subjective symptoms, clinical signs and presence of any restrictions on daily activities. Radiological follow-up: immediate postoperative plain X-rays was done to confirm the accurate position of pedicular screws and other $\mathrm{X}$-rays were done at the end of the follow-up (Fig. 6). An independent radiologist was invited to evaluate the status based on radiographic study. Surgical outcome was clas-

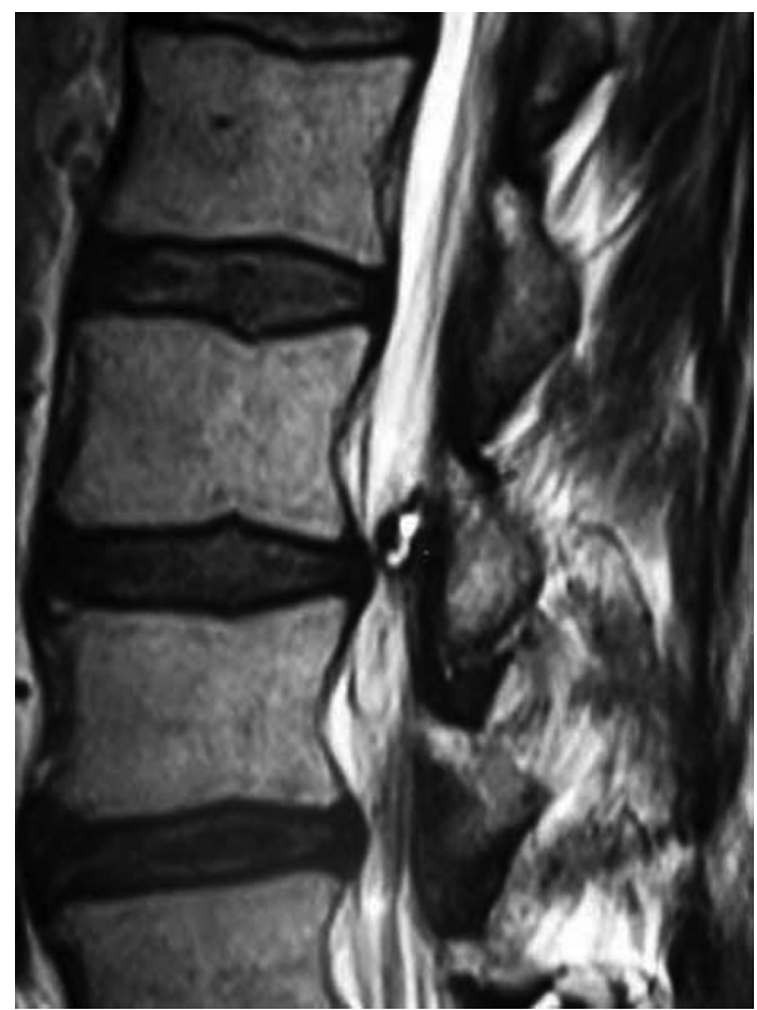

Fig. 2. A preoperative sagittal magnetic resonance imaging showing L4-5 synovial cyst.

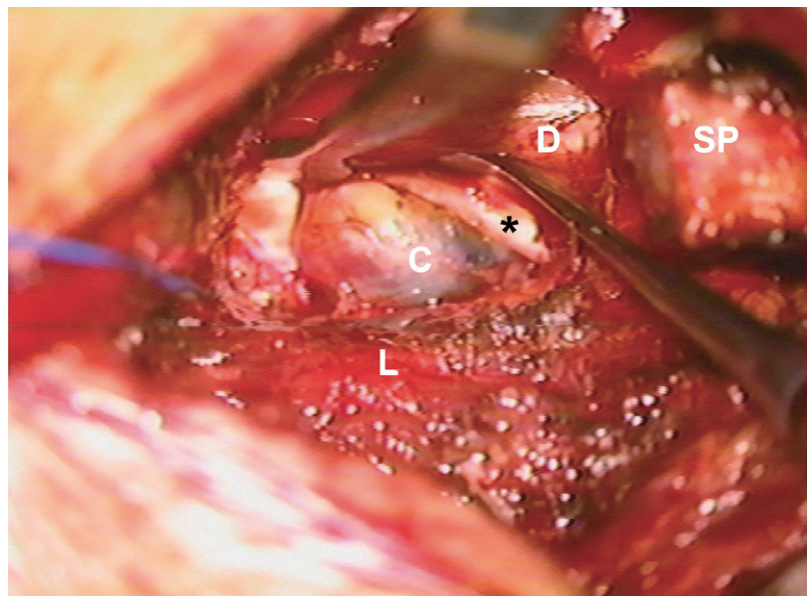

Fig. 3. Intraoperative photograph showing a large cystic mass filled with dark fluid. *, nerve root; $C$, cyst; D, dura; L, lamina; SP, spinous process. sified using Macnab's classification: excellent, complete relief of back/leg pain; good, occasional pain in back/leg; fair, some improvement in back/leg pain with occasional

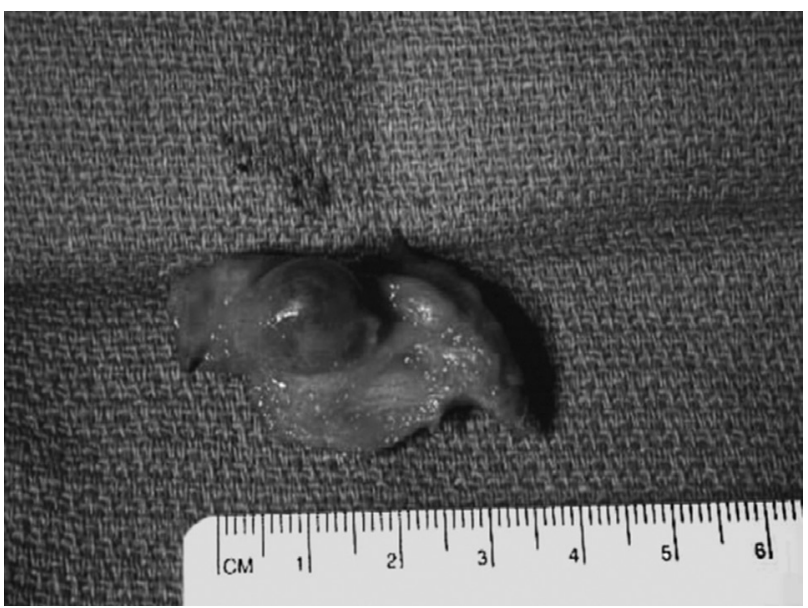

Fig. 4. Synovial cyst after complete excision.

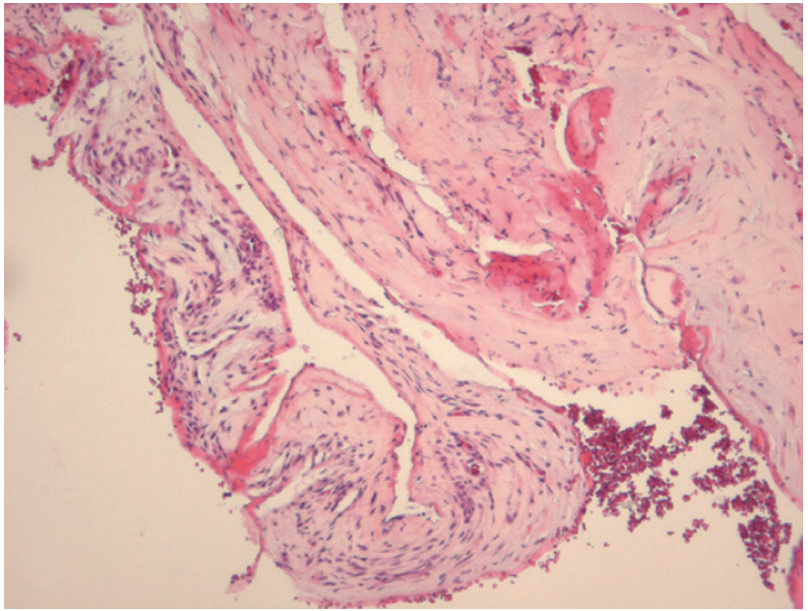

Fig. 5. Histological photo with $H \& E, \times 100$. stain showing thick fibromembranous tissue with no lining epithelium. The thick walls show some fibroblasts and mild myxoid stromal change.
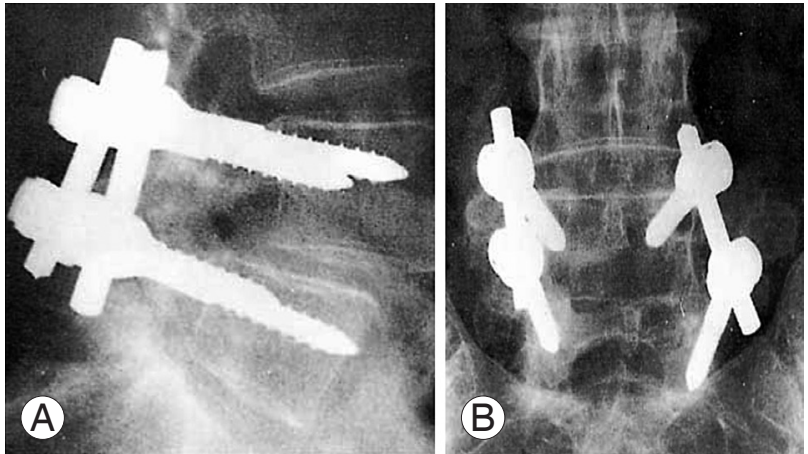

Fig. 6. (A, B) Postoperative X-ray (lateral, antero-posterior view) after laminectomy, excision of the cyst and transpedicular screw fixation. 
use of pain medications; poor, little or no change in pain or pain worse than before the surgery and a regular use of pain medications [7].

\section{Results}

Regarding the age distribution among 6 patients in our study, the peak incidence was between 35-45 years old in 3 patients ( $50 \%$ of total cases) and the least incidence was between $25-35$ years old in only one patient (16\% of total cases). The mean age was 40 years old. Most of patients in our study were males (4 patients, $66 \%$ ), while only 2 patients (34\%) were females with M:F ratio of 2:1 and this may be due to the nature of the heavy work males perform in the agricultural society and as a result have a higher likelihood of suffering trauma. The most common presenting feature in our study was sciatic pain in 4 patients (66\%), while sensory deficit was observed in only 2 patients (34\%). According to preoperative MRI (investigation of choice), the most common level affected was L4-5 in 5 patients (84\%), while only one patient (16\%) presented with an L3-4 synovial cyst. There was associated degenerative spondylolithesis in 4 patients (66\% of cases). The most frequent postoperative complications in our study were transient cerebro-spinal fluid (CSF) leakage, which was reported in 2 patients (34\%) and was managed conservatively by absolute bed rest, tight bandaging and frequent aspiration until complete resolution was seen and no collection was made till the end of the followup period. Superficial wound infection developed in only 1 patient (16\%) and was treated conservatively by bed rest, antibiotics and daily dressing change until complete healing. According to the results achieved in our study, the mean JOA score showed significant improvement as it increased from 6.5 on admission to 12.5 at the end of the study $(p<0.05)$. Regarding the outcome of patients in our study, it was excellent in 4 patients (66\% of cases) with complete resolution of preoperative symptoms and patients returning to their daily activities at the end of the follow up period, good in 2 patients (34\% of cases) with improvement of preoperative symptoms and had their sedentary life with restriction of heavy work.

\section{Discussion}

Synovial cysts are uncommon causes of neck pain, back pain, and radiculopathy. This pathology is more frequently located in the lumbar spine as was in our series and is very rare in the cervical and thoracic spine. Majority of synovial cysts occur in the lumbar spine (over 90\%), while cervical (under 5\%) and thoracic (under 5\%) lesions are encountered rarely. The most frequent location of synovial cyst for the lumbar level is L4-L5 as was in $84 \%$ of our patients [11]. The clinical presentation of a cyst depends on its volume, site, and relationship to the surrounding bony and neural structures. Cysts may be asymptomatic and found incidentally. Most of the symptomatic patients present with radicular pain and neurological deficits. In addition, clinical syndromes such as cauda equina, lateral recess, and spinal stenosis syndromes have been described [12].

In our study, all patients had lower back pain; with $66 \%$ having sciatic pain and $34 \%$ had associated neurogenic claudication. Associated sensory deficits were reported in $34 \%$ of patients included in the study. Our findings were consistent with reports found in other surgically treated cases in the literature [13]. CT scanning and MRI are the two neurodiagnostic imaging modalities recommended for characterization of synovial cysts and preoperative planning. The typical appearance of a cyst on a CT scan can be altered by its content, for example gas, calcification, blood, inflammation, and osseous structure involvement. On MRI, synovial cysts appear as well circumscribed, smooth, extradural in location, and adjacent to facet joints [14].

In our short study, there was associated grade I degenerative spondylolisthesis in 4 patients (66\% of cases) and lumbar canal stenosis in 2 patients (34\% of cases) and this was similar to Banning et al. [15] study where 12 of 19 patients (63\%) demonstrated spondylolisthesis while 6 (32\%) showed spinal canal stenosis. On the other hand, Lyons et al. [7]. fused 18 cases of 194 patients (9.2\%) with lumbar synovial cysts for documented preoperative instability based on preoperative MR and CT studies where cysts often extended into the yellow ligament underlying degenerated facet joints.

We were able to retrieve and confirm synovial tissue on histological examination. The distinction between synovial cysts (with a synovial lining) and ganglion cysts (without a lining) is a histological one. As other authors have discussed, there is no clinical relevance to differentiating between ganglion and synovial cysts, as their presentations, treatments, and prognosis are identical [16].

Conservative treatment modalities include bed rest, 
orthopedic corsets, CT-guided needle aspiration, and intra-articular injection of corticosteroid drugs. Few series have found that steroid injections provide acceptable long-term results. However, these treatments often show short term or no improvement at all. Surgical treatment is largely recommended in all cases of intractable pain or neurological deficit [17]. Current therapy for synovial cysts includes excision of the mass and lumbar decompression with or without fixation and fusion. In our study all patients underwent posterior decompression including wide laminectomy, removal of hypertrophied and often ossified yellow ligament in the lateral. Frequently the extreme lateral subarticular regions, superiorly, ventrally, and foraminally, the exiting nerve root and thecal sac were differentiated from the synovial cyst, which often fills the lateral gutter. Ipsilateral dissection with identification of the superior nerve root and thecal sac were followed, allowing for resection of the synovial cyst, often in a layered fashion: First, the cyst may be "gutted" or decompressed, removing the often thick, viscous contents. Second, it is ascertained whether or not a clean dural plane exists ventrally between the capsule of the cyst and underlying dura before excision of the cyst to avoid dural tear and CSF leakage. Fischgrund et al. [18] used the same technique where laminectomy was combined with posterior fixation at the segment of olisthy in his series, which included 67 patients with lumbar stenosis and degenerative spondylolisthesis.

All patients included in our study had excellent outcomes with improvement of preoperative manifestations and returning to normal daily activities during the follow up period. There were no major postoperative complications or neurological deficits except for only 2 cases who developed postoperative CSF leakage and were treated conservatively with strict bed rest, tight bandaging and systemic antibiotics and improved within 2 weeks. Our study correlated with that of Doyle and Merrilees [19] in their series of 194 patients that included cerebrospinal fluid fistula (3 patients), postoperative instability warranting secondary fusion (4/176 patients undergoing laminectomy), discitis (1 patient), epidural hematoma (1 patient), seroma (1 patient), phlebitis (1 patient), and death (1 patient from unrelated cardiac disease).

No recurrence was reported in our cases during the follow up period of 12 months postoperatively while Christophis et al. [20] observed that of their 31 patients with synovial cysts, recurrent cysts were observed in $3 \%$ of the cases and 3 patients (9\% of cases) required subsequent surgery including two spinal fusions.

\section{Conclusions}

Spinal cysts are commonly found at the L4-L5 level, the site of maximum instability. The etiology is still unclear, but underlying spinal instability has a strong association for formation of spinal cysts. Surgical resection and decompression with fusion remain an appropriate option. It appears that the optimal approach for patients with juxtafacet cysts remains unclear. Although this study included a small number of cases and we could not have statistically significant results, the good outcome of decompression of synovial cysts combined with posterior fixation and fusion without any major postoperative complications or recurrences encourage us to recommend this approach for patients with juxtafacet synovial cysts.

\section{Conflict of Interest}

No potential conflict of interest relevant to this article was reported.

\section{References}

1. Marshman LA, Benjamin JC, David KM, King A, Chawda SJ. "Disc cysts" and "posterior longitudinal ligament ganglion cysts": synonymous entities? Report of three cases and literature review. Neurosurgery 2005;57:E818.

2. Gazzeri R, Canova A, Fiore C, Galarza M, Neroni M, Giordano M. Acute hemorrhagic cyst of the ligamentum flavum. J Spinal Disord Tech 2007;20:536-8.

3. Metellus P, Flores-Parra I, Fuentes S, et al. A retrospective study of 32 lumbar synovial cysts: clinical aspect and surgical management. Neurochirurgie 2003;49:73-82.

4. Houten JK, Sanderson SP, Cooper PR. Spontaneous regression of symptomatic lumbar synovial cysts: report of three cases. J Neurosurg 2003;99:235-8.

5. Salmon B, Martin D, Lenelle J, Stevenaert A. Juxtafacet cyst of the lumbar spine: clinical radiological and therapeutic aspects in 28 cases. Acta Neurochir (Wien) 2001;143:129-34.

6. Onofrio BM, Mih AD. Synovial cysts of the spine. Neurosurgery 1988;22:642-7. 
7. Lyons MK, Atkinson JL, Wharen RE, Deen HG, Zimmerman RS, Lemens SM. Surgical evaluation and management of lumbar synovial cysts: the Mayo Clinic experience. J Neurosurg 2000;93:53-7.

8. Trummer M, Flaschka G, Tillich M, Homann CN, Unger F, Eustacchio S. Diagnosis and surgical management of intraspinal synovial cysts: report of 19 cases. J Neurol Neurosurg Psychiatry 2001;70:74-7.

9. Artico M, Cervoni L, Carloia S, Stevanato G, Mastantuono M, Nucci F. Synovial cysts: clinical and neuroradiological aspects. Acta Neurochir (Wien) 1997;139:176-81.

10. Eyster EF, Scott WR. Lumbar synovial cysts: report of eleven cases. Neurosurgery 1989;24:112-5.

11. Sandhu FA, Santiago P, Fessler RG, Palmer S. Minimally invasive surgical treatment of lumbar synovial cysts. Neurosurgery 2004;54:107-11.

12. Epstein NE. Lumbar synovial cysts: a review of diagnosis, surgical management, and outcome assessment. J Spinal Disord Tech 2004;17:321-5.

13. Cohen-Gadol AA, White JB, Lynch JJ, Miller GM, Krauss WE. Synovial cysts of the thoracic spine. J Neurosurg Spine 2004;1:52-7.

14. Shah RV, Lutz GE. Lumbar intraspinal synovial cysts: conservative management and review of the world's literature. Spine J 2003;3:479-88.

15. Banning CS, Thorell WE, Leibrock LG. Patient out- come after resection of lumbar juxtafacet cysts. Spine (Phila Pa 1976) 2001;26:969-72.

16. Ramieri A, Domenicucci M, Seferi A, Paolini S, Petrozza V, Delfini R. Lumbar hemorrhagic synovial cysts: diagnosis, pathogenesis, and treatment: report of 3 cases. Surg Neurol 2006;65:385-90.

17. Pirotte B, Gabrovsky N, Massager N, Levivier M, David P, Brotchi J. Synovial cysts of the lumbar spine: surgery-related results and outcome. J Neurosurg 2003;99:14-9.

18. Fischgrund JS, Mackay M, Herkowitz HN, Brower R, Montgomery DM, Kurz LT. 1997 Volvo Award winner in clinical studies. Degenerative lumbar spondylolisthesis with spinal stenosis: a prospective, randomized study comparing decompressive laminectomy and arthrodesis with and without spinal instrumentation. Spine (Phila Pa 1976) 1997;22:280712.

19. Doyle AJ, Merrilees M. Synovial cysts of the lumbar facet joints in a symptomatic population: prevalence on magnetic resonance imaging. Spine (Phila $\mathrm{Pa}$ 1976) 2004;29:874-8.

20. Christophis P, Asamoto S, Kuchelmeister K, Schachenmayr W. "Juxtafacet cysts", a misleading name for cystic formations of mobile spine (CYFMOS). Eur Spine J 2007;16:1499-505. 